(2) Open Access Full Text Article

ORIGINAL RESEARCH

\title{
Association Between Serum Human Epididymis Protein 4 Levels and Cardiovascular Events in Obese Patients with Breast Cancer
}

\author{
Li Wang' \\ $\mathrm{Nie} \mathrm{Hu}{ }^{1}$ \\ Zhongjun Jiang' \\ Ping Luo' \\ Nian $\mathrm{Yi}^{\mathrm{I}}$ \\ Yanhua Chen ${ }^{2}$ \\ Ning Zhou (iD) ${ }^{2}$ \\ Longfei Liu ${ }^{2}$
}

'Department of Breast Surgery, Nanhua Hospital, Nanhua University, Hengyang City, Hunan Province, 421002, People's Republic of China; ${ }^{2}$ Department of General Surgery, Nanhua Hospital, Nanhua University, Hengyang City, Hunan Province, 421002, People's Republic of China
Correspondence: Longfei Liu Department of General Surgery, Nanhua Hospital, Nanhua University, No. 336,

Dongfeng South Road, Hengyang City,

Hunan Province, 421002, People's

Republic of China

Tel +86 0734-8399028

Email liulongfei1977@aliyun.com
Background: Patients with cancer have a higher incidence of cardiovascular diseases (CVDs). We aimed to evaluate the relationship between serum human epididymal protein 4 (HE4) levels and cardiovascular events in obese patients with breast cancer.

Methods: Serum HE4 levels in 316 obese patients with breast cancer were measured at baseline and then prospectively followed up for approximately 36 months. The prognostic value of serum HE4 for predicting cardiovascular events was analyzed by the Cox proportional hazard model.

Results: Serum HE4 was significantly associated with CVD history after adjustment for confounding factors $(\mathrm{OR}=1.50 ; 95 \% \mathrm{CI} 1.23-3.43 ; \mathrm{P}=0.038)$ using multivariable logistic regression analysis. The multivariable Cox proportional hazard analysis suggested that serum HE4 had an independent prognostic value for predicting cardiovascular events in patients with breast cancer $(\mathrm{HR}=2.21,95 \%$ CI $1.60-5.13, P<0.001)$. Additionally, sensitivity analysis showed that the independent association still existed. Stratified analysis showed that this relationship was not affected by chemoradiotherapy.

Conclusion: Serum HE4 is significantly related to cardiovascular events and poses good prognostic value for predicting cardiovascular events in obese patients with breast cancer. Serum HE4 may be a valuable indicator for the early detection of cardiovascular complications in obese patients with breast cancer.

Keywords: human epididymis protein 4, radiotherapy, breast cancer, cardiovascular events, prognostic value, obese

\section{Introduction}

Breast cancer and cardiovascular diseases (CVDs) are the important contributors to the causes of chronic disease worldwide. ${ }^{1}$ The number of comorbidities will rise with aging populations and enhanced therapies.,3 Emerging evidence suggests a close relationship between breast cancer and CVDs due to shared risk factors, including aging, tobacco, alcohol, obesity, low levels of physical activity, diabetes, hyperlipidemia and poor dietary composition, ${ }^{4-8}$ and common pathogeneses such as inflammation and oxidative stress. ${ }^{9-12}$ Radiotherapy and chemotherapy for breast cancer can significantly increase the risk of CVDs. ${ }^{13-17}$ Importantly, cardiovascular events are an important complication of radiotherapy in female patients with breast cancer. ${ }^{13-15}$ Finding ways to predict and prevent cardiovascular injury in patients with breast cancer has become an intensely studied topic in cardiovascular medicine in recent decades. The early detection of cardiovascular complications in patients with breast cancer will provide good chance to improve prognosis. 
Human epididymis protein 4 (HE4) is a $20-25 \mathrm{kDa}$ glycoprotein that can be found on membranes of cells and the cytoplasm and in circulating blood18,19. Apart from being overexpressed in cancers such as ovarian cancer and endometrial cancer, ${ }^{20-23} \mathrm{HE} 4$ has a significant role in immune defense. ${ }^{22-26}$ Cancer itself and chemoradiotherapy can accelerate the progression of the inflammatory response and contribute to an elevated risk of cardiovascular complications. ${ }^{9-12}$ Based on the role of HE4 in the inflammatory process, ${ }^{24-26}$ it was hypothesized that HE4 might be linked to cardiovascular events in patients with breast cancer.

To date, however, few studies have investigated the association of serum HE4 with cardiovascular events in patients with breast cancer. We aimed to evaluate whether elevated HE4 levels contribute to a higher risk of cardiovascular events after confounding factors were adjusted in patients with breast cancer. This was the first study to investigate the prognostic value of serum HE4 in predicting cardiovascular events in patients with breast cancer.

\section{Methods}

\section{Study Sample}

A total of 316 hospitalized patients with breast cancer from Affiliated Hospital of Nanhua University in China who had undergone chemoradiotherapy between February 2015 and November 2016 were included. After standardized chemoradiotherapy, all included patients with breast cancer were stable clinically and then were followed up by phone prospectively. The diagnosis of breast cancer was performed by 2 tumor experts using the combination of pathological examination and clinical features. ${ }^{27}$ Some patients were excluded because of other types of cancers or other serious diseases. Ten patients with breast cancer were excluded from the study due to other malignant diseases $(\mathrm{N}=2)$ or severe liver $(\mathrm{N}=5)$ and lung diseases $(\mathrm{N}=3)$. Additionally, to evaluate the serum levels of HE4 in patients with breast cancer, 359 healthy subjects without malignancies and other severe illnesses were selected as the healthy controls (Table 1).

Variables related to demographic characteristics and lifestyle were collected from medical records and patient interviews. Cardiovascular disease (CVD) history, such as hypertension, coronary heart disease (CHD), stroke, diabetes mellitus and others, was obtained from the patient's previous medical records. According to the Declaration of Helsinki guidelines, the Ethics Committee of Nanhua
Hospital of Nanhua University approved this study, and written informed consent was collected from all included patients.

\section{Follow-Up}

All included patients with breast cancer after discharge were followed up for a median of 3 years (4-42 months) prospectively. Patients were contacted by telephone review four times a year until the occurrence of cardiovascular events (endpoints) during the follow-up period. To achieve the purpose of the study, cardiovascular events were defined as heart failure, myocardial infarct, stroke, and sudden cardiac death during the follow-up period. The diagnosis of cardiovascular events was performed by two cardiovascular experts. Our reliable and continuous surveillance can ensure adequate collection of cardiovascular events. As long as one of these events occurred, the patient's follow-up was completed. If current status of these patients was uncertain, referring cardiologists and/or general practitioners were contacted and invited to identify the patient's condition.

\section{Detection of Serum HE4}

Fasting venous blood from all included patients with breast cancer was collected on the first morning after admission. Fasting venous blood was immediately processed by centrifugation and then processed to test HE4 levels. Electrochemiluminescence immunoassay (Cobas e 601, F. Hoffmann-La Roche Ltd.) was used to test serum HE4 levels. HE4 levels over 1500 pmol/L samples were measured again (measurement range, $15-1500 \mathrm{pmol} /$ $\mathrm{L}$; coefficient of variation of precision $<5 \%$; detection limit, $5 \mathrm{pmol} / \mathrm{L}$ ).

\section{Laboratory Measurements}

Venous blood was collected from all included patients with breast cancer on the first morning after admission and tested for hemoglobin $(\mathrm{Hb})$, hs-CRP (hs-C reactive protein), albumin (ALB), low-density lipoprotein (LDL), high-density lipoprotein (HDL), fasting blood glucose (FBG) and glycosylated hemoglobin (HbAlc) with the use of the Siemens ADVIA 2400 automatic biochemistry analyzer (Siemens AG). The estimated glomerular filtration rate (eGFR) was calculated by the Modification of Diet in Renal Disease (MDRD) formula. ${ }^{28}$ 
Table I Clinical Characteristics in 316 Female Patients with Breast Cancer at Baseline

\begin{tabular}{|c|c|c|c|}
\hline Variables & $\begin{array}{l}\text { Patients with Breast Cancer } \\
\qquad(n=3 \mid 6)\end{array}$ & Healthy Subjects $(n=359)$ & $P$ value \\
\hline Age (years) & $64.7(57.9-76.8)$ & $65.2(55.9-74.3)$ & 0.682 \\
\hline BMI $\left(\mathrm{kg} / \mathrm{m}^{2}\right)$ & $30.2(27.9-32.3)$ & $29.2(26.4-32.1)$ & $<0.001$ \\
\hline Current smoker, n (\%) & $21(6.3)$ & $23(6.4)$ & 0.962 \\
\hline Current drinker, n (\%) & $32(10.1)$ & $28(7.8)$ & 0.067 \\
\hline Admission systolic BP (mmHg) & $137.5(122.6-154.8)$ & $135.2(120.3-150.6)$ & 0.013 \\
\hline Admission diastolic BP (mmHg) & $84.5(80.2-92.8)$ & $81.3(78.5-90.3)$ & 0.037 \\
\hline Chemoradiotherapy, n (\%) & $316(100)$ & - & - \\
\hline First chemoradiotherapy, n (\%) & $114(21.8)$ & - & - \\
\hline Chemoradiotherapy ( $\geq 2), n$ (\%) & $247(78.2)$ & - & - \\
\hline \multicolumn{4}{|l|}{ CVD history } \\
\hline Hypertension, n (\%) & $221(69.9)$ & $108(30.1)$ & $<0.001$ \\
\hline Coronary heart disease, n (\%) & $56(17.7)$ & $27(7.5)$ & $<0.001$ \\
\hline Stroke, n (\%) & $52(16.5)$ & $24(6.7)$ & $<0.001$ \\
\hline Diabetes mellitus, n (\%) & $101(31.9)$ & $67(18.7)$ & $<0.001$ \\
\hline Others, n (\%) & $12(3.8)$ & $5(1.4)$ & $<0.001$ \\
\hline \multicolumn{4}{|c|}{$\begin{array}{l}\text { Cardiovascular events of the follow-up } \\
\text { period }\end{array}$} \\
\hline Miocardial infarction, n (\%) & $10(3.2)$ & - & - \\
\hline Stroke, n (\%) & $4(1.3)$ & - & - \\
\hline Heart failure, n (\%) & $6(1.9)$ & - & - \\
\hline Sudden cardiac death, n (\%) & $2(0.6)$ & - & - \\
\hline \multicolumn{4}{|l|}{ Laboratory measurements } \\
\hline $\mathrm{Hb}(\mathrm{g} / \mathrm{L})$ & $108(93-115)$ & $119(113-124)$ & 0.003 \\
\hline $\operatorname{ALB}(g / L)$ & $33.5(31.6-39.5)$ & $36.7(32.5-45.7)$ & $<0.001$ \\
\hline eGFR $\left(\mathrm{mL} / \mathrm{min} / 1.73 \mathrm{~m}^{2}\right)$ & $56.3(54.5-58.8)$ & $59.7(57.9-61.6)$ & 0.008 \\
\hline $\mathrm{Hs}-\mathrm{CRP}(\mathrm{pg} / \mathrm{mL})$ & $7.5(2.3-11.9)$ & $7.96(2.4-12.6)$ & 0.055 \\
\hline $\mathrm{HDL}(\mathrm{mmol} / \mathrm{L})$ & $1.21 \pm 0.11$ & $1.32 \pm 0.13$ & 0.340 \\
\hline LDL (mmol/L) & $2.63 \pm 1.12$ & $2.56 \pm 1.20$ & 0.191 \\
\hline FBG $(\mathrm{mmol} / \mathrm{L})$ & $6.8 . \pm 1.5$ & 5.3. \pm 1.3 & 0.014 \\
\hline $\mathrm{HbAlc}(\%)$ & $6.4(5.5-8.6)$ & $5.4(4.7-8.2)$ & 0.019 \\
\hline HE4 (pmol/L) & $|32.4 \pm| 4.8$ & $66.5 \pm 11.2$ & $<0.001$ \\
\hline
\end{tabular}

Notes: Data are presented as mean \pm SD for normally distributed data, as median (interquartile range) for nonnormally distributed data, and as $\mathrm{n}$ (\%) for categorical variables.

Abbreviations: BMI, body mass index; BP, blood pressure; CVD, cardiovascular disease; eGFR, estimated glomerular filtration rate; Hb, hemoglobin; hs-CRP, hs-C reactive protein; ALB, albumin; FBG, fasting blood glucose on admission; HbAlc, glycosylated hemoglobin; HDL, high density lipoprotein; LDL, low density lipoprotein; HE4, human epididymal protein 4 .

\section{Statistical Analyses}

The Mann-Whitney $U$-test was performed to analyze data that were not normally distributed, and the data are presented as the median (interquartile range [IQR]). Independent t-tests were performed to assess normally distributed data, which are expressed as the mean $\pm \mathrm{SD}$. Categorical variables were analyzed by using the chi- square test. First, logistic regression analysis was performed to assess the association between serum levels of HE4 and CVD history in patients with breast cancer at baseline after controlling for age, body mass index (BMI), current smoker, current drinker, admission systolic and diastolic BP and laboratory measurements. Then, the Cox proportional hazard model was used to identify the 
Table 2 Multivariate Logistic Regression Analysis for Association of Serum HE4 Levels with CVD History in Patients with Breast Cancer at Baseline

\begin{tabular}{|l|c|c|c|}
\hline Variables & Model I & Model 2 & Model 3 \\
\hline Serum HE4 (pmol/L) & & & 1.000 (ref.) \\
Quartile I $(\leq 83.5 \mathrm{pmol} / \mathrm{L})$ & 1.000 (ref.) & 1.000 (ref.) & $1.27(1.07-2.97)$ \\
Quartile 2 $(83.5-132.4 \mathrm{pmol} / \mathrm{L})$ & $1.42(1.10-3.22)$ & $1.35(1.09-3.12)$ & $1.31(1.10-3.00)$ \\
Quartile 3 $(132.4-201.6 \mathrm{pmol} / \mathrm{L})$ & $1.52(1.23-3.33)$ & $1.42(1.12-3.22)$ & $1.50(1.23-3.43)$ \\
Quartile 4 ( $\geq 201.6 \mathrm{pmol} / \mathrm{L})$ & $1.76(1.32-3.94)$ & $1.65(1.26-3.50)$ & 0.038 \\
\hline P-trend & 0.012 & 0.025 & 0 \\
\hline
\end{tabular}

Notes: Model I: No adjustment. Model 2: Adjusted for age, BMI, current smoker, current drinker, admission systolic and diastolic BP. Model 3: Adjusted for age, BMI, current smoker, current drinker, admission systolic and diastolic BP and laboratory measurements.

Abbreviations: HE4, human epididymal protein 4; CVD, cardiovascular disease; BMI, body mass index; BP, blood pressure.

independent prognostic value of serum HE4 for predicting cardiovascular events in patients with breast cancer during the follow-up period after controlling for these same confounders. Additionally, sensitivity and stratification analyses were used to evaluate the association of serum HE4 levels with cardiovascular events by adding "CVD history" and "chemoradiotherapy" as covariates. SPSS 25.0 was used for all of the analyses in our study. $P \leq 0.05$ was considered to be statistically significant.

\section{Results}

\section{Clinical Characteristics of All Included Patients with Breast Cancer After Admission ( $\mathrm{N}=316)$}

To evaluate the serum levels of HE4 in patients with breast cancer, 359 healthy subjects without malignancies and other severe illnesses were selected as the healthy controls. We found that patients with breast cancer had significantly higher serum levels of HE4 than healthy controls (Table 1). The clinical characteristics of patients with breast cancer are also detailed in Table 1 . The median age of all included patients with breast cancer was 64.7 years. The number of cardiovascular events during the follow-up period, including myocardial infarction, stroke, heart failure and sudden cardiac death, was 10 (3.2\%), $4(1.3 \%), 6(1.9 \%)$ and $2(0.6 \%)$ in patients with breast cancer, respectively.

\section{Elevated HE4 Levels Were Independently Linked to CVD History After Admission}

To assess the association of serum HE4 levels with CVD history in patients with breast cancer, a logistic regression model was used (Table 2). Model 1 showed that elevated HE4 levels were related to a higher risk of CVD history $(\mathrm{OR}=1.762,95 \%$ CI 1.318-3.940, $\mathrm{P}=0.012)$ when no adjustment was made. After adjustments for age, BMI, current smoker, current drinker, and admission systolic and diastolic BP were made, the results of Model $2(\mathrm{OR}=1.653,95 \% \mathrm{CI}$ 1.263-3.502, $\mathrm{P}=0.025$ ) were consistent with those of Model 1. The independent relationship was significant and minimally changed after continuing to add laboratory measurements to Model 2 ( $\mathrm{OR}=1.502,95 \%$ CI $1.228-3.432, \quad \mathrm{P}=0.038$; Model 3).

\section{Cox Proportional Hazard Model for Association Between Serum HE4 and Cardiovascular Events in 316 Patients with Breast Cancer}

All included patients with breast cancer $(\mathrm{N}=316)$ were prospectively followed up for a median period of 36 months (range $=4-42$ ). The total number of cardiovascular events that occurred was $34(10.8 \%)$ in all included patients with breast cancer. To assess the prognostic performance of serum HE4 in predicting composite events, multivariable Cox proportional hazard regression analyses were used (Table 3). A multivariable Cox proportional hazard model suggested that HE4 $(\mathrm{HR}=2.207,95 \%$ CI 1.602-5.132, $P<0.001)$ may be considered a valuable prognostic factor for predicting cardiovascular events in patients with breast cancer after adjustments for age, BMI, current smoker, current drinker, admission systolic and diastolic BP and laboratory measurements were made. The Kaplan-Meier model showed that patients with serum HE4 levels above the mean had an increased possibility of cardiovascular events compared with patients with HE4 levels below the mean value (Figure 1).

Additionally, we performed a sensitivity analysis to assess the association between serum HE4 and cardiovascular events in these patients by adding "CVD history" as a covariate (Table 4). We found that elevated HE4 levels 
Table 3 Cox Proportional Hazard Analysis for Predicting Cardiovascular Events in 316 Patients with Breast Cancer

\begin{tabular}{|c|c|c|c|}
\hline Variables & Model I & Model 2 & Model 3 \\
\hline \multicolumn{4}{|l|}{ Serum HE4 (pmol/L) } \\
\hline Quartile I ( $\leq 83.5$ pmol/L) & 1.000 (ref.) & 1.000 (ref.) & 1.000 (ref.) \\
\hline Quartile 2 (83.5-132.4 pmol/L) & $1.92(|.31-3.7|)$ & $1.74(1.22-3.13)$ & $1.57(1.12-3.00)$ \\
\hline Quartile 3 (I32.4-201.6 pmol/L) & $2.25(1.63-5.35)$ & $2.17(1.53-4.57)$ & $2.01(1.43-4.01)$ \\
\hline Quartile 4 ( $\geq 201.6$ pmol/L) & $2.46(1.81-6.55)$ & $2.37(1.74-5.33)$ & $2.21(1.60-5.13)$ \\
\hline$P$-trend & $<0.001$ & $<0.001$ & $<0.001$ \\
\hline
\end{tabular}

Notes: Model I: No adjustment. Model 2: Adjusted for age, BMI, current smoker, current drinker, admission systolic and diastolic BP. Model 3: Adjusted for age, BMI, current smoker, current drinker, admission systolic and diastolic BP and laboratory measurements.

Abbreviations: HE4, human epididymal protein 4; CVD, cardiovascular disease; BMI, body mass index; BP, blood pressure.

were still linked to an increased risk of cardiovascular events. Stratified analysis suggested that the significant association between HE4 levels and cardiovascular events in patients with breast cancer was not affected by adding "chemoradiotherapy" as a covariate (Table 5).

\section{Discussion}

Our findings demonstrated that HE4 may be a valuable prognostic factor for predicting cardiovascular events in patients with breast cancer using multivariable Cox proportional hazard analysis.

The common risk factors for cancer and CVDs include age, sex, smoking, drinking, BMI $>25$, lack of exercise, unreasonable diet structure, abnormal increases in blood glucose, blood lipids and others. ${ }^{4-8}$ Cancer can also increase the risk of CVDs or aggravate the original CVDs by increasing common risk factors. ${ }^{8}$ Similarly, reducing the risk factors for CVDs can reduce the risk of cancer. Studies have also shown that the tumor itself is a risk factor for CVDs. ${ }^{9-12}$ For example, an increased number of macrophages in leukemia can promote the progression of atherosclerosis. ${ }^{9,10}$ Anticancer drugs can retard the progression of CVDs to a certain extent. Anti-CVD drugs (such as aspirin tablets) can have a certain inhibitory effect on some malignant tumors that is associated with common mechanisms of cancer and CVDs, such as chronic inflammation and oxidative stress. ${ }^{9-12}$ Importantly, cardiotoxicity

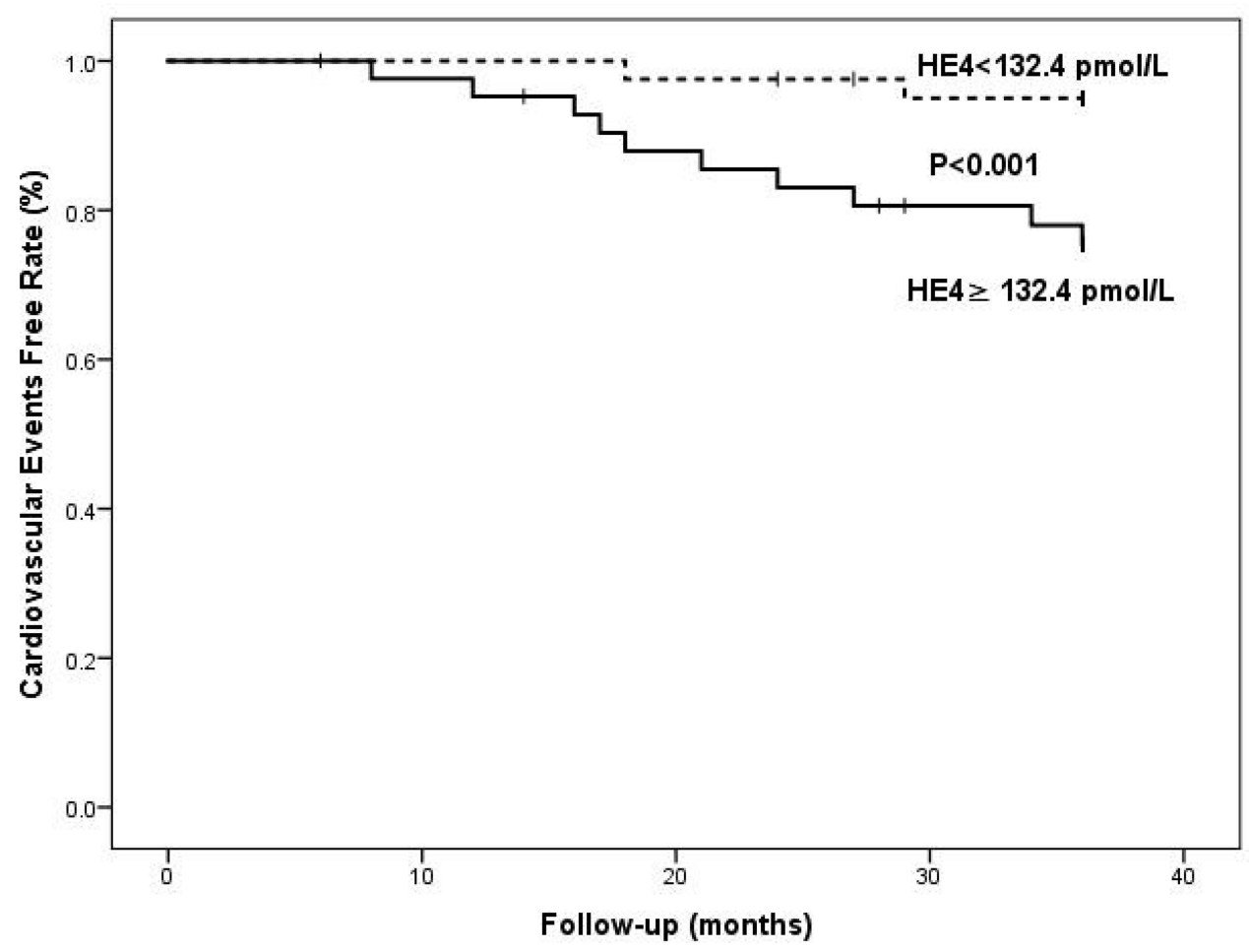

Figure I Kaplan-Meier analysis of cardiovascular events free stratified into 2 groups by the mean levels of serum human epididymis protein 4 (HE4). 
Table 4 Cox Proportional Hazard Analysis for Predicting Cardiovascular Events in 316 Patients with Breast Cancer by Sensitivity Analysis

\begin{tabular}{|l|c|c|c|}
\hline Variables & Model I & Model 2 & Model 3 \\
\hline Serum HE4 (pmol/L) & & & 1.000 (ref.) \\
Quartile I $(\leq 83.5 \mathrm{pmol} / \mathrm{L})$ & $1.000(\mathrm{ref})$ & $1.000(\mathrm{ref})$ & $1.52(\mathrm{I}) \mathrm{II}-2.94)$ \\
Quartile 2 $(83.5-132.4 \mathrm{pmol} / \mathrm{L})$ & $1.81(1.3 \mathrm{I}-3.60)$ & $1.70(1.21-3.43)$ & $1.98(1.34-4.33)$ \\
Quartile 3 $(\mathrm{I} 32.4-201.6 \mathrm{pmol} / \mathrm{L})$ & $2.13(1.53-5.55)$ & $2.05(1.49-4.93)$ & $2.14(1.50-5.03)$ \\
Quartile 4 $(\geq 201.6 \mathrm{pmol} / \mathrm{L})$ & $2.37(\mathrm{I}) 62-6.35)$ & $2.28(1.57-5.42)$ & $<0.001$ \\
\hline P-trend & $<0.001$ & $<0.001$ & \\
\hline
\end{tabular}

Notes: Model I: CVD history. Model 2: Adjusted for age, BMI, current smoker, current drinker, admission systolic and diastolic BP and CVD history. Model 3: Adjusted for age, BMI, current smoker, current drinker, admission systolic and diastolic BP, laboratory measurements and CVD history.

Abbreviations: HE4, human epididymal protein 4; CVD, cardiovascular disease; BMI, body mass index; BP, blood pressure.

caused by anticancer therapy is a major cause of morbidity or death in patients with cancer. Radiotherapy and chemotherapy for cancer can cause cardiovascular side effects, causing acute or chronic cardiovascular complications, or aggravating preexisting CVDs. For example, patients with a history of heart disease are more vulnerable to radiation-induced heart damage than those without a history of heart disease. ${ }^{13-15}$ Therefore, the early prediction of cardiovascular complications in patients with cancer might provide a change to improve prognosis.

In the present study, we found first that serum HE4 had an independent association with CVD history and cardiovascular events during the 3-year follow-up in patients with breast cancer after admission, which can be partly explained by the mechanistic research performed in previous studies. ${ }^{29-31}$ Previous evidence has suggested that the inflammatory state caused by cancer and cancer-related treatment promotes cardiovascular events. ${ }^{29-31}$ The elevated HE4 levels might be the result of amplification of the inflammatory response in patients with cancer, which is consistent with our hypothesis and results. Additionally, several studies have shown that elevated HE4 levels are associated with renal dysfunction caused by chronic kidney disease. ${ }^{32-34}$ The eGFR of our included patients was normal, so the impact of renal function abnormalities on our results was excluded. Studies have also reported that HE4 is linked with ovarian, cervical, and lung cancer. ${ }^{20,21}$ To eliminate the impact of the diseases on this study, all included patients with these diseases were excluded after admission. Our results implied that HE4 may be considered a highly valuable biomarker for the early detection of cardiovascular events in patients with breast cancer.

Several strengths exist in this study. We first showed that serum HE4 might be a valuable prognostic factor for cardiovascular events in patients with breast cancer. In addition, rigorous adjudication of cardiovascular events and comprehensive follow-up was carried out to ensure the reliability of our results. Finally, the measurement of serum HE4 is both analytically stable (coefficient of variation of precision $<5 \%$ ) and widely available, making it a convenient indicator for clinicians to analyze.

Of course, several limitations should be noted in the current study. First, the results are originated from a single-center study with a small sample size. Future studies need to be performed

Table 5 Cox Proportional Hazard Analysis for Predicting Cardiovascular Events in 316 Patients with Breast Cancer by Stratified Analysis

\begin{tabular}{|l|c|c|c|}
\hline Variables & Model I & Model 2 & Model 3 \\
\hline $\begin{array}{c}\text { Chemoradiotherapy ( } \geq 2) \\
\text { HE4 (per I-SD increase) }\end{array}$ & $2.48(1.72-6.57)$ & $2.25(1.66-5.57)$ & $2.20(1.52-5.08)$ \\
P value & $<0.001$ & $<0.001$ & $<0.001$ \\
\hline $\begin{array}{l}\text { First chemoradiotherapy } \\
\text { HE4 (per I-SD increase) }\end{array}$ & $1.95(1.350-4.98)$ & $1.73(1.33-4.40)$ & $1.64(1.27-4.00)$ \\
P value & 0.001 & 0.003 & 0.010 \\
\hline
\end{tabular}

Notes: Model I: CVD history. Model 2: Adjusted for age, BMI, current smoker, current drinker, admission systolic and diastolic BP and CVD history. Model 3: Adjusted for age, BMI, current smoker, current drinker, admission systolic and diastolic BP, laboratory measurements and CVD history. Abbreviations: HE4, human epididymal protein 4; CVD, cardiovascular disease; BMI, body mass index; BP, blood pressure. 
to assess the value of serum HE4 for predicting cardiovascular events in patients with breast cancer. Second, considering the close relationships of serum HE4 and chronic kidney disease and many malignant tumors, the results of this study are not applicable to all populations with these diseases. Whether the relationship between HE4 and cardiovascular events is caused by renal function has not been fully analyzed, although eGFR, an important marker of renal function, was corrected in our regression model. Third, sufficient confounding factor correction may also lead to overfitting of the correction model, which can make the results of the study slightly inconsistent with the actual results. Fourth, only one time of serum HE4 measurement was performed in all included patients at baseline. Other variables that may affect serum HE4 levels are unknown. Only one serum test may not fully represent the real level of HE4 in these patients, leading causing some bias to our results.

\section{Conclusions}

Serum HE4 might be a valuable prognostic factor for early detection of cardiovascular events in obese patients with breast cancer. HE4 may be a reliable indicator to assist clinicians in predicting cardiovascular events in patients with breast cancer.

\section{Funding}

The study was supported by the Scientific Research Project of Hunan Health Commission (20201948) and the Natural Science Foundation of Hunan Province (2020JJ4552).

\section{Disclosure}

The authors report no conflicts of interest in this work.

\section{References}

1. Hooper L, Thompson RL, Harrison RA, et al. Risks and benefits of omega 3 fats for mortality, cardiovascular disease, and cancer: systematic review. BMJ. 2006;332(7544):752-760. doi:10.1136/ bmj.38755.366331.2F

2. Khaw KT, Dowsett M, Folkerd E, et al. Endogenous testosterone and mortality due to all causes, cardiovascular disease, and cancer in men european prospective investigation into cancer in Norfolk (EPIC-Norfolk) Prospective Population Study. Circulation. 2007;116 (23):2694-2701. 7.719005 doi:10.1161/CIRCULATIONAHA.10

3. Omenn GS, Goodman GE, Thornquist MD, et al. Effects of a combination of beta carotene and vitamin $\mathrm{A}$ on lung cancer and cardiovascular disease. $N$ Engl J Med. 1996;2(18):1150-1155. doi:10.1056/NEJM199605023341802

4. Romo ML, Mccrillis AM, Brite J, et al. Pharmacologic androgen deprivation and cardiovascular disease risk factors: a systematic review. Eur J Clin Invest. 2015;45.
5. Foraker R, Abdelrasoul M, Kuller L, et al. Abstract MP08: cardiovascular health and incident cardiovascular disease and cancer. Circulation. 2013;AMP8.

6. Carlos MB, Mario G-B, Jorge G-A, et al. Iron: protector or risk factor for cardiovascular disease? Still controversial. Nutrients. 2013;5 (7):2384-2404. doi:10.3390/nu5072384

7. Konety S, Prizment AE, Blaes A, et al. Shared risk factors in cardiovascular disease and cancer. Circulation. 2016.

8. Koene RJ, Prizment AE, Blaes A, et al. Shared risk factors in cardiovascular disease and cancer. Circulation. 2016;133 (11):1104-1114. doi:10.1161/CIRCULATIONAHA.115.020406

9. Deng FE, Shivappa N, Tang Y, et al. Association between diet-related inflammation, all-cause, all-cancer, and cardiovascular disease mortality, with special focus on prediabetics: findings from NHANES III. Eur J Nutr. 2017;56(3):1085-1093. doi:10.1007/ s00394-016-1158-4

10. Yoshikazu N, Komuro I. Gab docking proteins in cardiovascular disease, cancer, and inflammation. Int J Inflam. 2013.

11. Agnieszka S. Novel therapeutic approaches in limiting oxidative stress and inflammation. Curr Pharm Biotechnol. 2012.

12. RD Khanna, Karki K. Inflammation, free radical damage, oxidative stress and cancer. Interdiscip $J$ Microinflammation. 2014;1(1). doi:10.4172/ijm.1000109

13. Nielsen KM, Offersen BV, Nielsen HM, et al. Short and long term radiation induced cardiovascular disease in patients with cancer. Clin Cardiol. 2017;40(4):255-261. doi:10.1002/clc.22634

14. Boekel NB, Schaapveld M, Gietema JA, et al. OC-0259: radiotherapy/chemotherapy-related cardiovascular disease in breast cancer patients: a population-based study. Radiother Oncol. 2013;106:S101-S101. doi:10.1016/S0167-8140(15)32565-2

15. Boerman L, Berendsen AJ, van der Meer P, et al. Long-term follow-up for cardiovascular disease after chemotherapy and/or radiotherapy for breast cancer in an unselected population. Supp Care Cancer. 2014. doi:10.1007/s00520-014-2156-9

16. Travis LB, Ng AK, Allan JM. Second malignant neoplasms and cardiovascular disease following radiotherapy. JNCI. 2012;104 (5):357-370. doi:10.1093/jnci/djr533

17. Argirovic D, Jelicradosevic L, Argirovic A. C48 The long-term side effects of adjuvant radiotherapy vs carboplatin chemotherapy in clinical stage A seminomatous testicular tumors. Eur Urol Suppl. 2009;8(8):674. doi:10.1016/S1569-9056(09)75073-0

18. Kirchhoff C, Habben I, Ivell R, et al. A major human epididymis-specific cDNA encodes a protein with sequence homology to extracellular proteinase inhibitors. Biol Reprod. 1991;45 (2):350-357. doi:10.1095/biolreprod45.2.350

19. Kirchhoff C. Molecular characterization of epididymal proteins. Rev Reprod. 1998;3(2):86-95. doi:10.1530/ror.0.0030086

20. Hellstrom I, Raycraft J, Hayden-Ledbetter M, et al. The HE4 (WFDC2) protein is a biomarker for ovarian carcinoma. Cancer Res. 2003;63(13):3695-3700.

21. Plebani M. HE4 in gynecological cancers: report of a European investigators and experts meeting. Clin Chem Lab Med. 2012;50 (12):2127-2136. doi: $10.1515 / \mathrm{cclm}-2012-0373$

22. Hertlein L, Stieber P, Kirschenhofer A, et al. Human epididymis protein 4 (HE4) in benign and malignant diseases. Clin Chem Lab Med. 2012;50(12):2181-2188. doi:10.1515/cclm-2012-0097

23. Galgano MT, Hampton GM, Frierson HJ. Comprehensive analysis of HE4 expression in normal and malignant human tissues. Mod Pathol. 2006;19(6):847-853. doi:10.1038/modpathol.3800612

24. Bingle CD, Vyakarnam A. Novel innate immune functions of the whey acidic protein family. Trends Immunol. 2008;29(9):444-453. doi:10.1016/j.it.2008.07.001

25. Escudero JM, Auge JM, Filella X, et al. Comparison of serum human epididymis protein 4 with cancer antigen 125 as a tumor marker in patients with malignant and nonmalignant diseases. Clin Chem. 2011;57(11):1534-1544. doi:10.1373/clinchem.2010.157073 
26. Drapkin R, von Horsten HH, Lin Y, et al. Human epididymis protein 4 (HE4) is a secreted glycoprotein that is overexpressed by serous and endometrioid ovarian carcinomas. Cancer Res. 2005;65(6):2162S154. doi:10.1158/0008-5472.CAN-04-3924

27. Spittle M, Morgan D. Women remain confused about breast cancer. BMJ. 2019;7183:600.

28. Levey AS, Stevens LA, Schmid CH, et al. A new equation to estimate glomerular filtration rate. Ann Intern Med. 2009;150 (9):604-612. doi:10.7326/0003-4819-150-9-200905050-00006

29. Figueroa AL, Abdelbaky A, Macnabb MH, et al. Abstract 16509: increased arterial inflammation by FDG-PET-CT is associated with an increased risk of cardiovascular events among obese low-intermediate risk individuals. Circulation. 2012;(21):A16509.

30. Duprez DA, Otvos J, Tracy RP, et al. High-density lipoprotein subclasses and noncardiovascular, noncancer chronic inflammatory-related events versus cardiovascular events: the multi-ethnic study of atherosclerosis. J Am Heart Assoc. 2015;4(9):e002295. doi:10.1161/JAHA.115.002295
31. Duprez DA, Otvos J,Sanchez O, et al. Comparison of the predictive value of GlycA and other biomarkers of inflammation for total death, incident cardiovascular events, noncardiovascular and noncancer inflammatory-related events, and total cancer events. Clin Chem. 2016.

32. Yuan T, Li Y. Human epididymis protein 4 as a potential biomarker of chronic kidney disease in female patients with normal ovarian function. Lab Med. 2017;48(3):238-243. doi:10.1093/labmed/lmx036

33. Huang Y, Jiang H, Zhu L. Human epididymis protein 4 as an indicator of acute heart failure in patients with chronic kidney disease. Lab Med. 2020;51(2):169-175.

34. Nagy B, Krasznai ZT, Balla H, et al. Elevated human epididymis protein 4 concentrations in chronic kidney disease. Ann Clin Biochem. 2012;49(4):377-380. doi:10.1258/acb.2011.011258

\section{Publish your work in this journal}

Diabetes, Metabolic Syndrome and Obesity: Targets and Therapy is an international, peer-reviewed open-access journal committed to the rapid publication of the latest laboratory and clinical findings in the fields of diabetes, metabolic syndrome and obesity research. Original research, review, case reports, hypothesis formation, expert opinion and commentaries are all considered for publication. The manuscript management system is completely online and includes a very quick and fair peer-review system, which is all easy to use. Visit http://www.dovepress.com/testimonials.php to read real quotes from published authors. 